\title{
Exposure to low dose computed tomography for lung cancer screening and risk of cancer: secondary analysis of trial data and risk-benefit analysis
}

\author{
Cristiano Rampinelli, ${ }^{1}$ Paolo De Marco, ${ }^{2}$ Daniela Origgi, ${ }^{3}$ Patrick Maisonneuve, ${ }^{4}$ Monica Casiraghi, \\ Giulia Veronesi, ${ }^{5,6}$ Lorenzo Spaggiari, ${ }^{5,7}$ Massimo Bellomi ${ }^{1,7}$
}

${ }^{1}$ Department of Medical Imaging and Radiation

Sciences, European Institute of Oncology, Milan, Italy

${ }^{2}$ Medical Physics School, University of Milan, Milan, Italy

${ }^{3}$ Division of Medical Physics,

European Institute of Oncology, Milan, Italy

${ }^{4}$ Division of Epidemiology and Biostatistics, European Institute of Oncology, Milan, Italy

${ }^{5}$ Division of Thoracic Surgery, European Institute of Oncology, Milan, Italy

${ }^{6}$ Division of Thoracic Surgery, Humanitas Research Hospital, Rozzano, Italy

${ }^{7}$ Department of Oncology and Hematology-Oncology,

University of Milan, Milan, Italy

Correspondence to:

C Rampinelli

cristiano.rampinelli@ieo.it

Cite this as: $B M J$ 2017:356:i347

http://dx.doi.org/10.1136/bmj.j347

Accepted: 29 December 2016

\section{ABSTRACT}

OBJECTIVE

To estimate the cumulative radiation exposure and lifetime attributable risk of cancer incidence associated with lung cancer screening using annual low dose computed tomography (CT).

DESIGN

Secondary analysis of data from a lung cancer screening trial and risk-benefit analysis.

SETTING

10 year, non-randomised, single centre, low dose CT, lung cancer screening trial (COSMOS study) which took place in Milan, Italy in 2004-15 (enrolment in 2004-05). Secondary analysis took place in 2015-16.

\section{PARTICIPANTS}

High risk asymptomatic smokers aged 50 and older, who were current or former smokers ( $\geq 20$ pack years), and had no history of cancer in the previous five years.

\section{MAIN OUTCOME MEASURES}

Cumulative radiation exposure from low dose $\mathrm{CT}$ and positron emission tomography (PET) CT scans, calculated by dosimetry software; and lifetime attributable risk of cancer incidence, calculated from the Biological Effects of lonizing Radiation VII (BEIR VII) report.

RESULTS

Over 10 years, 5203 participants (3439 men, 1764 women) underwent 42228 low dose CT and 635 PET CT scans. The median cumulative effective dose at the 10th year of screening was $9.3 \mathrm{mSv}$ for men and 13.0

\section{WHAT IS ALREADY KNOWN ON THIS TOPIC}

Lung cancer is the leading cause of cancer death among both men and women

Screening of high risk individuals with low dose computed tomography reduces

lung cancer mortality by $20 \%$ if compared with chest radiography

Excess cancer risks related to ionising radiation from low dose computed

tomography are a major concern in lung cancer screening

\section{WHAT THIS STUDY ADDS}

The median cumulative radiation exposure from low dose computed tomography screening over 10 years was $9.3 \mathrm{mSv}$ for men and $13.0 \mathrm{mSv}$ for women

The lifetime attributable risk of major cancers from low dose computed tomography screening ranged from 2.6 to 8.1 major cancers per 10000 participants, according to participant age and sex

One radiation induced cancer would be expected in every 108 lung cancers detected after 10 years of computed tomography screening

Radiation exposure from low dose computed tomography and the risk of radiation induced cancer can be considered acceptable in light of the substantial mortality reduction associated with lung cancer screening

mSv for women. According to participants' age and sex, the lifetime attributable risk of lung cancer and major cancers after 10 years of $\mathrm{CT}$ screening ranged from 5.5 to 1.4 per 10000 people screened, and from 8.1 to 2.6 per 10000 people screened, respectively. In women aged 50-54, the lifetime attributable risk of lung cancer and major cancers was about fourfold and threefold higher than for men aged 65 and older, respectively. The numbers of lung cancer and major cancer cases induced by 10 years of screening in our cohort were 1.5 and 2.4 , respectively, which corresponded to an additional risk of induced major cancers of $0.05 \%$ (2.4/5203). 259 lung cancers were diagnosed in 10 years of screening; one radiation induced major cancer would be expected for every 108 (259/2.4) lung cancers detected through screening.

\section{CONCLUSION}

Radiation exposure and cancer risk from low dose CT screening for lung cancer, even if non-negligible, can be considered acceptable in light of the substantial mortality reduction associated with screening.

\section{Introduction}

Lung cancer is the leading cause of cancer for both men and women in the USA. In 2015, the American Cancer Society expected more than 200000 new cases of lung cancer, about $14 \%$ of the total new cancers, with a five year survival rate of $18 \%{ }^{1}$

The US National Lung Screening Trial has shown that screening of high risk populations with low dose computed tomography (CT) reduces lung cancer mortality by more than $20 \%$ when compared with chest radiography. ${ }^{2}$ As a consequence, several medical societies currently recommend CT lung screening, and in the USA, the Centers for Medicare and Medicaid Services have granted a positive insurance coverage decision on screening. ${ }^{3}$ Millions of healthy high risk individuals are therefore theoretically eligible for CT lung cancer screening.

There is concern, however, that exposure to the ionising radiation of low dose CT in lung cancer screening might increase the risk of developing solid cancers and leukaemia. ${ }^{4}$ The concrete existence of this increased risk as related to low dose radiation (doses $<50 \mathrm{mSv}$ ) is controversial, ${ }^{5-7}$ although this topic deserves additional consideration because ionising radiation from CT screening is delivered to healthy people.

So far, there is limited knowledge of the levels of radiation exposure in lung cancer screening regarding both cumulative radiation exposure and the associated cancer risk, particularly in long term studies. Brenner ${ }^{8}$ 
estimated that if $50 \%$ of all current and former smokers in the US population aged 50-75 received annual CT screening, the number of lung cancers associated with radiation from screening would be about 36000 , a $1.8 \%$ (95\% confidence interval 0.5 to 5.5 ) increase over the otherwise expected number. McCunney and $\mathrm{Li}^{9}$ reported that lung screening participants could experience a cumulative exposure to ionising radiation over 20-30 years, which exceeds the lifetime dose experienced by nuclear power workers and atomic bomb survivors. The results of these studies cannot be considered conclusive, however, because they are based on the assumption of an arbitrary, pre-established radiation dose for all individuals, regardless of sex, age, or body size.

The aim of this study was to retrospectively evaluate the cumulative radiation exposure and lifetime attributable risk of cancer incidence associated with low dose CT from a 10 year lung cancer screening programme.

\section{Methods}

All data reported in the present study were retrospectively assembled and analysed from a 10 year, non-randomised, observational, lung cancer screening trial (COSMOS study)..$^{11}$ In brief, 5203 asymptomatic high risk participants (age $>50$ and smoking history with $\geq 20$ pack years, and no history of cancer in the past five years) underwent annual low dose CT for 10 consecutive years. Additional recalls for suspicious findings were performed with low dose CT scans and positron emission tomography (PET) CT scans, according to the study design. The COSMOS study took place in Milan, Italy, in 2004-15 (enrolment in 2004-05). A comprehensive description of the COSMOS study, as well as low dose CT protocols and PET CT scans, are reported in the supplementary material. The present study (that is, the secondary analysis), took place in 2015-16.

\section{Radiation exposure from CT screening}

To consider the overall radiation exposure in the population, the annual repeated low dose CT scans and the follow-up low dose CT scans of each patient were collected for each year of screening. In addition, all the PET CT scans performed within the study were considered to evaluate the cumulative exposure dose. At the end of the 10th year of CT screening, all performed examinations were collected from the radiology information-picture archiving and communication system (RIS-PACS) and sent to Radimetrics (Bayer Healthcare), a commercially available software for monitoring and tracking patient radiation exposure. ${ }^{12}$ Organ doses and effective doses were retrospectively estimated by Radimetrics for each low dose CT examination and for the CT acquisitions of the PET CT scans. Data and scanning parameters were collected from CT examinations, and patients were matched-according to age, sex, weight, and dimension-to six and five different adult phantoms for women and men, respectively. This size specific calculation of organ doses is more accurate than calculating from the standard reference patient used by other software.

Organ doses are calculated by use of a look-up table based on MonteCarlo simulations for the selected phantoms, ${ }^{13}$ and are used to estimate effective dose according to weighting factors from the International Commission on Radiological Protection. ${ }^{14}$ For PET CT scans, the contribution of the radioactive tracer to organ doses and effective doses is calculated by the absorbed dose coefficients per unit activity administered $(\mathrm{mGy} / \mathrm{MBq}) .^{15}$

Total estimated organ dose and effective dose for one patient were calculated as the sum of the doses of each low dose CT examination (screening rounds performed plus recalls) and PET CT scans when performed. Because cumulative organ and effective doses were non-normally distributed, median and range were considered.

\section{Cancer risk estimation}

For each age, sex, and organ, we determined the lifetime attributable risk of cancer incidence from a 100 $\mathrm{mSv}$ organ equivalent dose table 12D-1 of the National Research Council's Biological Effects of Ionizing Radiation VII (BEIR VII) report. ${ }^{4}$ When missing, age specific lifetime attributable risk was estimated by polynomial interpolation of lifetime attributable risk values reported for ages 50,60, 70, and 80. This lifetime attributable risk from a theoretical $100 \mathrm{mSv}$ organ dose was scaled linearly on the basis of the actual organ dose measured at each CT scan. Lifetime attributable risk was calculated for each of the 42228 low dose CT scans performed during the COSMOS trial and added together to calculate the lifetime attributable risk for the entire COSMOS population.

\section{Patient involvement}

No patients were involved in setting the research question or the outcome measures, nor were they involved in developing plans for design or implementation of the study. No patients were asked to advise on interpretation or writing up of results. There are no plans to disseminate the results of the research to study participants or the relevant patient community.

\section{Results}

\section{Radiation exposure from CT screening}

During the 10 years of the COSMOS lung cancer screening trial, 5203 high risk participants underwent 42228 low dose CT examinations, including 39981 annual CT scans, 1965 first recall CT scans, and 282 second recall CT scans for lung cancer screening. As part of the screening protocol, 635 PET CT scans were performed in 522 participants with suspicious findings (table 1). A total of 259 lung cancers were diagnosed after 10 years of CT screening.

The median effective dose delivered at the baseline screening round was $1.0 \mathrm{mSv}$ (range 0.6-16.5) for men and $1.4 \mathrm{mSv}$ (0.9-14.9) for women. Median cumulative effective doses from both low dose CT and PET CT scans at the third, fifth, and 10th year of screening were 3.0 $\mathrm{mSv}$ (1.9-27.4), 5.2 mSv (2.9-39.6), and 9.3 mSv (5.6-42.7) for men, respectively; and $4.2 \mathrm{mSv}$ (2.9-23.3), $7.2 \mathrm{mSv}$ (4.1-26.8), and $13.0 \mathrm{mSv}$ (8.0-33.5) for women, respectively (table 2). A PET CT scan delivered an additional 
median radiation exposure of $4.0 \mathrm{mSv}$ (1.2-28.8). Complete information on the effective doses and organ specific exposition doses from low dose CT scans only and from low dose CT scans plus PET CT scans are provided in appendix 2 (web tables S1 and S2).

Overall, 15805 examinations were performed with eight detector row CT scans, 22132 with 16 detector row CT scans, and 4291 with 64 detector row CT scans. Average effective doses for one low dose CT examination for the three CT scanners were $1.07 \mathrm{mSv}$ (standard deviation 0.29), $1.05 \mathrm{mSv}$ (0.27), and $0.64 \mathrm{mSv}$ (0.15), respectively.

\section{Estimated risk of cancer from CT screening}

Table 3 shows lung cancers detected after 10 years of CT screening and lifetime attributable risk of lung cancer and major cancers by sex and age of COSMOS trial participants. The lifetime attributable risk of cancer incidence was extrapolated from the BEIR VII report to estimate lifetime attributable risks of lung cancer after

\begin{tabular}{|c|c|c|c|c|}
\hline $\begin{array}{l}\text { Screening } \\
\text { round }\end{array}$ & $\begin{array}{l}\text { No of annual } \\
\text { CT scans }\end{array}$ & $\begin{array}{l}\text { No (\%) of first } \\
\text { recall CT } \\
\text { scans per year }\end{array}$ & $\begin{array}{l}\text { No (\%) of second } \\
\text { recall CT scans } \\
\text { per year }\end{array}$ & $\begin{array}{l}\text { No (\%) of PET } \\
\text { CT scans per } \\
\text { year }\end{array}$ \\
\hline Baseline & 5203 & $482(9.3)$ & $75(1.4)$ & $160(3.1)$ \\
\hline 2nd year & 4822 & $142(2.9)$ & $23(0.5)$ & $68(1.4)$ \\
\hline 3rd year & 4583 & $198(4.3)$ & $32(0.7)$ & $74(1.6)$ \\
\hline 4th year & 4380 & $251(5.7)$ & $47(1.1)$ & $62(1.4)$ \\
\hline 5th year & 4120 & $213(5.2)$ & $35(0.8)$ & $66(1.6)$ \\
\hline 6th year & 3856 & $197(5.1)$ & $24(0.6)$ & $62(1.6)$ \\
\hline 7th year & 3654 & $170(4.7)$ & $16(0.4)$ & $41(1.1)$ \\
\hline 8th year & 3449 & 118 (3.4) & $14(0.4)$ & $35(1.0)$ \\
\hline 9th year & 3182 & $103(3.2)$ & $10(0.3)$ & $41(1.3)$ \\
\hline 10th year & 2732 & $91(3.3)$ & $6(0.2)$ & $26(1.0)$ \\
\hline Total No of scans & 39981 & 1965 & 282 & 635 \\
\hline
\end{tabular}

$\mathrm{CT}=$ computed tomography; $\mathrm{PET}=$ positron emission tomography.
10 years of CT screening. This lifetime attributable risk of lung cancer after 10 years of CT screening ranged between 5.5 per 10000 participants ( 1 in 1811) for women starting screening at age 50-54, and 1.4 per 10000 participants ( 1 in 6908) for a man starting screening aged 65 and older. In the same two groups of women and men, lifetime attributable risks of major cancers ranged between 8.1 per 10000 participants ( 1 in 1229) and 2.6 per 10000 participants (1 in 3898), respectively (table 3 ).

Based on our exposure data applied to tables in the BEIR VII report, we estimated the number of lung cancers and major cancers induced by 10 years of low dose CT screening to be 1.5 and 2.4, respectively, corresponding to a theoretical risk of induced major cancer of $0.05 \%$ (2.4/5203). Compared with the number of lung cancers detected over 10 years, one radiation induced lung cancer would be expected for every 173 (259/1.5) lung cancers diagnosed, and one radiation induced major cancer would be expected for every 108 (259/2.4) lung cancers detected through screening.

The lifetime attributable risk of lung cancer was estimated to be about four times greater for women aged 50-54 years than for older men aged 65 and older (5.5 v 1.4 per 10000 participants). However, the lifetime attributable risk of major cancers was three times higher in the corresponding groups (8.1 $v 2.6$ per 10000 participants), reflecting differences in age and sex risk reported in the BEIR VII tables.

Figure 1 shows the estimated number of lung and major cancers induced by radiation for men and women, for 10000 people screened. As expected, the risk of developing radiation induced cancer was higher for women at all ages, and the risk decreased in both sexes while the age of exposure increased. There were always fewer than five radiation induced cancers per 10000 people screened in men, and fewer than 10 per 10000 people screened in women.

\begin{tabular}{|c|c|c|c|c|c|c|c|c|}
\hline & \multicolumn{4}{|l|}{ Men } & \multicolumn{4}{|l|}{ Women } \\
\hline & Baseline & 3rd year & 5th year & 10th year & Baseline & 3rd year & 5 th year & 10th year \\
\hline No of participants & 3439 & 3056 & 2768 & 1850 & 1764 & 1527 & 1352 & 884 \\
\hline Effective dose (mSv) & 1.0 & 3.0 & 5.2 & 9.3 & 1.4 & 4.2 & 7.2 & 13.0 \\
\hline \multicolumn{9}{|l|}{ Organ dose (mGy): } \\
\hline Breast & - & - & - & - & 2.5 & 7.6 & 13.0 & 23.3 \\
\hline Bladder & 0.0 & 0.1 & 0.1 & 0.2 & 0.0 & 0.1 & 0.1 & 0.2 \\
\hline Colon & 0.2 & 0.7 & 1.2 & 2.2 & 0.2 & 0.6 & 1.1 & 2.0 \\
\hline Oesophagus & 1.4 & 4.5 & 7.7 & 13.6 & 1.8 & 5.6 & 9.5 & 16.9 \\
\hline Gallbladder & 1.5 & 4.6 & 7.9 & 14.0 & 1.3 & 4.2 & 7.2 & 12.9 \\
\hline Heart & 2.1 & 6.8 & 11.5 & 20.5 & 2.5 & 7.6 & 13.0 & 23.2 \\
\hline Kidney & 1.9 & 5.9 & 10.1 & 18.0 & 1.8 & 5.6 & 9.7 & 17.4 \\
\hline Liver & 1.9 & 6.1 & 10.4 & 18.4 & 2.1 & 6.6 & 11.2 & 20.0 \\
\hline Lung & 2.3 & 7.1 & 12.2 & 21.7 & 2.7 & 8.3 & 14.2 & 25.3 \\
\hline Ovaries & - & - & - & - & 0.1 & 0.2 & 0.3 & 0.6 \\
\hline Marrow & 0.8 & 2.5 & 4.3 & 7.6 & 0.9 & 2.8 & 4.7 & 8.4 \\
\hline Skeleton & 1.4 & 4.3 & 7.4 & 13.3 & 1.7 & 5.3 & 9.1 & 16.5 \\
\hline Spleen & 2.0 & 6.1 & 10.5 & 18.6 & 2.2 & 6.8 & 11.7 & 20.9 \\
\hline Stomach & 1.9 & 5.9 & 10.0 & 17.9 & 2.0 & 6.1 & 10.4 & 18.7 \\
\hline Thyroid & 0.2 & 0.6 & 1.1 & 1.9 & 0.5 & 1.6 & 2.8 & 5.2 \\
\hline Uterus & - & - & - & - & 0.1 & 0.2 & 0.3 & 0.5 \\
\hline
\end{tabular}




\begin{tabular}{|c|c|c|c|c|}
\hline $\begin{array}{l}\text { Participant age } \\
\text { and sex at start of } \\
\text { screening }\end{array}$ & $\begin{array}{l}\text { No of } \\
\text { participants }\end{array}$ & $\begin{array}{l}\text { No of lung } \\
\text { cancers } \\
\text { detected }\end{array}$ & $\begin{array}{l}\text { No of estimated } \\
\text { radiation induced lung } \\
\text { cancers (LAR/10000) }\end{array}$ & 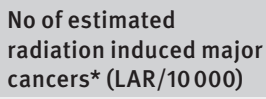 \\
\hline \multicolumn{5}{|l|}{$50-54$} \\
\hline Male & 1153 & 35 (1 in 33) & $0.24(2.1)$ & $0.43(3.7)$ \\
\hline Female & 606 & 19 (1 in 32) & $0.33(5.5)$ & $0.49(8.1)$ \\
\hline \multicolumn{5}{|l|}{$55-59$} \\
\hline Male & 1114 & 56 (1 in 20) & $0.21(1.9)$ & $0.38(3.4)$ \\
\hline Female & 611 & 31 (1 in 20) & $0.31(5.1)$ & $0.44(7.2)$ \\
\hline \multicolumn{5}{|l|}{$60-64$} \\
\hline Male & 716 & 54 (1 in 13) & $0.12(1.7)$ & $0.22(3.0)$ \\
\hline Female & 345 & 13 (1 in 27) & $0.16(4.5)$ & $0.21(6.2)$ \\
\hline \multicolumn{5}{|l|}{$\geq 65$} \\
\hline Male & 456 & 41 (1 in 11) & $0.07(1.4)$ & $0.12(2.6)$ \\
\hline Female & 202 & $10(1$ in 20$)$ & $0.08(3.8)$ & $0.10(5.1)$ \\
\hline All ages, both sexes & 5203 & 259 detected & 1.5 induced & 2.4 induced \\
\hline
\end{tabular}
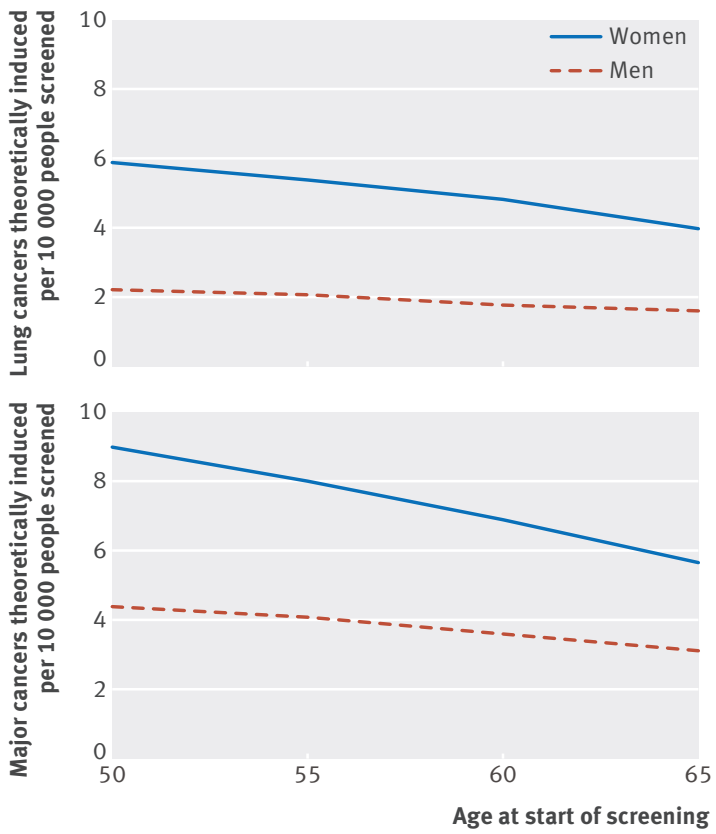

Fig 1 | Lung cancers and major cancers theoretically induced per 10000 people screened, according to sex and age at start of $\mathrm{CT}$ screening for lung cancer

\section{Discussion}

\section{Principal findings}

In this study, we showed that the median cumulative effective dose after 10 years of CT screening is roughly 9 $\mathrm{mSv}$ for men and $13 \mathrm{mSv}$ for women. By comparison with other diagnostic CT examinations, this means that an individual participating in a 10 year screening programme of low dose CT would receive a dose similar to that delivered to another undergoing one standard CT scan to the chest (7-8 mSv) or abdomen-pelvis (13-14 $\mathrm{mSv}) .{ }^{1617}$ Furthermore, if we consider that the 10 year average dose from background sources in the USA is about $30 \mathrm{mSv}$, we can assume that 10 years of screening delivers only a third of the exposure to natural background radiation in the same period. ${ }^{1819}$

Our study estimates that, after 10 years of low dose CT screening in 5203 asymptomatic high risk patients (age $>50$ and smoking history $\geq 20$ pack years) with no history of cancer in the past five years, 1.5 lung cancers and 2.4 major cancers were theoretically induced by radiation. This corresponds to an additional overall risk of major cancer of $0.05 \%$ (2.4 in 5203 screened people). When compared with the number of cancers detected by CT screening in the same period, one major cancer is theoretically induced by radiation for roughly every 100 detected.

As expected, the lifetime attributable risk for women was greater than for men at all ages, with a relative risk up to four times greater for lung cancer and up to three times greater for major cancers. This difference is related to the increased radiosensitivity of women compared with men and to the risk of breast cancer associated with chest CT scans. ${ }^{4}$

\section{Strengths and weaknesses of the study}

In this study, we analysed radiation exposure data assembled from a cohort of patients who were enrolled in a 10 year CT screening programme. To our knowledge, this is the first analysis in a lung cancer screening population where a specific dose has been calculated for each of the 42228 low dose CT scans-overcoming the limitation of using a fixed radiation exposure for all participants, as seen in previous studies.

One strength of this study was the dosimetry calculation, which was provided by an advanced software program (Radimetrics) for each low dose CT scan. Although not a patient specific dosimetry, this software can calculate organ doses and hence effective doses for six groups of women and five groups of men, according to their dimensions. Therefore, this size specific calculation of organ doses provides greater accuracy than software that uses only the standard reference patient for its calculations. In this case, with a fixed acquisition protocol, we could observe no difference in organ and effective dose calculations. Variations in organ doses among participants were also related to the number of low dose CT scans and PET CT scans received during the study and to the different doses delivered by the three CT scanners.

The assessment of cancer risk according to the BEIR committee is based on the linear no-threshold model and on data collected from environmental, occupational, and medical studies, and from atomic bomb survivors. The risk estimates of the BEIR VII report are thus based on risk models generated from studies on people exposed to high levels of radiation, and they are extrapolated to low doses using the linear no-threshold model for radiation risk. Various authors have discussed the weaknesses of the BEIR VII report, focusing on the linear no-threshold model ${ }^{72021}$ and on uncertainties in the translation of risk from high doses and dose rates in the Japanese population to lower doses and dose rates in the US population.

A segment of the scientific community claims that there is a threshold for low dose radiation carcinogenesis, 
and warns against quantitative estimation of health risks that might be too small to be observed or are non-existent below $50 \mathrm{mSv} .^{22-24}$ However, so far there is not sufficient evidence to suggest that the risk from radiation exposure is non-existent below a certain dose. Therefore, the linear no-threshold model stands as a precautionary recommendation that follows a conservative approach. Furthermore, all the risk estimates in the tables of the BEIR VII report have been obtained as a consensus opinion of a committee, and the inferred risk at lower doses probably overestimates the risk of cancer induction. ${ }^{25}$

To estimate the risk of cancer in individuals, the optimum approach would be to use specific organ doses and hence age and sex adjusted coefficients. ${ }^{26}$ The effective dose has been implemented mainly for protection purposes, and its use in medical practice as a measure of individual risk goes beyond its intended purpose. ${ }^{1427}$ Another factor to consider is that our results are related to the specific COSMOS study design, and different patient selection (eg, by age or smoking status) might lead to dissimilar results in terms of cancer risk.

As well as study population, screening nodule management has substantial implications for the overall radiation exposure and cancer risk. In fact, different thresholds of nodule size, interval follow-up, and the use of PET CT scans are determinant sources of variations. More conservative guidelines, as suggested by the American College of Radiology (Lung-RADS version $1.0),{ }^{28}$ could lead to lower population doses.

\section{Comparison with other studies}

In the National Lung Screening Trial, the effective dose estimates were $1.6 \mathrm{mSv}$ and $2.4 \mathrm{mSv}$ for one low dose CT scan for men and women, respectively. ${ }^{29}$ Our results show an effective dose at a baseline of $1.0 \mathrm{mSv}$ for men and $1.4 \mathrm{mSv}$ for women. These doses are $40 \%$ lower than in the National Lung Screening Trial, also considering the additional dose delivered by PET CT scans performed in our study. Study design, scanning parameters, and calculation methods might account for these differences. The scanning parameters at a typical study site in the National Lung Screening Trial are similar to those of our baseline data. However, the calculation method of organ dose and effective dose of the National Lung Screening Trial is based on software that does not take into account the patient's body size. The estimated organ dose in the trial was $4.9 \mathrm{mGy}$ to the breast, and nearly $5 \mathrm{mGy}$ to the lungs for both men and women. By comparison, taking into account the patient's body size, we found a dose of $2.5 \mathrm{mGy}$ to the breast, and doses of $2.3 \mathrm{mGy}$ and $2.7 \mathrm{mGy}$ to the lungs for men and women, respectively. This difference between these results highlights how the estimates of organ and effective doses with software that takes into account the patient's body size is an important source of variability.

The cumulative effective dose for the National Lung Screening Trial over three years was $4.8 \mathrm{mSv}$ for men and $7.2 \mathrm{mSv}$ for women. In the same period, we found a cumulative effective dose of $3.0 \mathrm{mSv}$ for men and 4.2
mSv for women (table 2). In our study, the cumulative effective dose, and the consequent risk of cancer, was the sum of radiation exposure both from low dose CT scans and PET CT scans. The contribution of one PET CT scan was $4.0 \mathrm{mSv}$, compared with the 10 year low dose CT cumulative exposure of $9.2 \mathrm{mSv}$ and $12.9 \mathrm{mSv}$ for men and women, respectively (table S2). Therefore, screening studies that do not include PET CT scans in their protocols could lead to a lower radiation exposure. The ITALUNG screening trial, after four rounds of screening, reported that $77.4 \%$ of the delivered dose was from annual low dose CT scans and $22.6 \%$ from further investigations (fluorodeoxyglucose-PET and CT guided biopsy). ${ }^{30}$ Further investigations assessing the role of study design in measuring radiation exposure are therefore needed.

Accurate risk prediction models can now quantify an individual's risk of developing or dying from lung cancer, and help identify people at high enough risk to undergo screening. ${ }^{31} 32$ Little is known, however, about the additional risk of cancer caused by exposure to radiation from screening itself. In a risk evaluation based on an assumed dose to the lung of $5.2 \mathrm{mGy}$, Brenner ${ }^{8}$ estimated an additional lung cancer risk of $1.8 \%$ caused by annual lung CT screening. Our results, conversely, show a theoretical additional risk of $0.05 \%$ (2.4 cases of major cancers induced in 5203 people).

\section{Further considerations and conclusions}

Even if our results show that cumulative radiation exposure after 10 years of low dose CT screening is substantially limited, there are still possibilities for further reduction. An accurate patient selection can substantially reduce the radiation exposure of low risk individuals, and the definition of an accurate study design is essential for the improvement of the diagnostic flowchart, minimising unnecessary radiation exposure.

New CT scanners and optimised acquisitions protocols can reduce the dose by up to $40 \%$, as seen in the differences in effective doses among 8, 16, and 64 slice scanners reported in our results. In addition, according to our protocol, CT images were reconstructed by use of the standard filtered back projection. With the introduction of a new iterative reconstruction algorithm, it is now possible to achieve the same diagnostic image quality with a dose that is reduced by up to $80 \%$ compared with standard filtered back projection. ${ }^{33-36}$

Another consideration is the detection of incidental findings on low dose CT scans. ${ }^{3738}$ New findings could lead to additional radiation exposure through further testing. In the present study, we did not consider the additional exposure from examinations performed for collateral findings, resulting in a slight underestimation of overall cancer risk.

In conclusion, radiation exposure and cancer risk from CT screening, even if non-negligible, can be considered acceptable in light of the substantial mortality reduction associated with screening.

Contributors: CR and MB conceived and designed the study. PDM and DO acquired and analysed the data. PM carried out the statistical analysis. All authors drafted and revised the manuscript. All authors 
have read and approved the final manuscript. CR and MB are the study guarantors and are responsible for the decision to submit for publication.

Funding: The COSMOS study was supported by the Italian Association for Cancer Research. The study sponsor was not involved in the study design, collection, analysis, and interpretation of data, or the writing of the article or the decision to submit it for publication. The authors were independent from study sponsor. The secondary analysis was not funded.

Competing interests: All authors have completed the ICMJE uniform disclosure form at www.icmje.org/coi disclosure.pdf and declare: support from the Italian Association for Cancer Research for the COSMOS study; no financial relationships with any organisations that might have an interest in the submitted work in the previous three years; no other relationships or activities that could appear to have influenced the submitted work.

Ethical approval: Not required.

Data sharing: No additional data available.

The lead author affirms that the manuscript is an honest, accurate, and transparent account of the study being reported; that no important aspects of the study have been omitted; and that any discrepancies from the study as planned have been explained.

This is an Open Access article distributed in accordance with the Creative Commons Attribution Non Commercial (CC BY-NC 4.0) license, which permits others to distribute, remix, adapt, build upon this work non-commercially, and license their derivative works on different terms, provided the original work is properly cited and the use is non-commercial. See: http://creativecommons.org/licenses/ by-nc/4.0/

1 Siegel RL, Miller KD, Jemal A. Cancer statistics, 2015. CA Cancer J Clin 2015;65:5-29. doi:10.3322/caac.21254.

2 Aberle DR, Adams AM, Berg CD, et al. National Lung Screening Trial Research Team. Reduced lung-cancer mortality with low-dose computed tomographic screening. N Engl J Med 2011;365:395-409. doi:10.1056/NEJMoa1102873.

3 Centers for Medicare \& Medicaid Services. Decision memo for screening for lung cancer with low dose computed tomography (LDCT). (CAG-00439N). 2015. https://www.cms.gov/medicarecoverage-database/details/nca-decision-memo.aspx?NCAld=274.

4 National Research Council. Health risks from exposure to low levels of ionizing radiation: BEIR VII-phase 2.National Academies Press, 2006.

Hendee WR, O'Connor MK. Radiation risks of medical imaging: separating fact from fantasy. Radiology 2012;264:312-21. doi:10.1148/radiol.12112678.

6 Brenner DJ, Hall EJ. Cancer risks from CT scans: now we have data, what next?Radiology 2012:265:330-1. doi:10.1148/radiol.12121248.

7 Calabrese EJ, O'Connor MK. Estimating risk of low radiation doses - a critical review of the BEIR VII report and its use of the linear no-threshold (LNT) hypothesis. Radiat Res 2014;182:463-74. doi:10.1667/RR13829.1.

8 Brenner DJ. Radiation risks potentially associated with low-dose CT screening of adult smokers for lung cancer. Radiology 2004;231:440 5. doi:10.1148/radiol.2312030880.

9 McCunney RJ, Li J. Radiation risks in lung cancer screening programs: a comparison with nuclear industry workers and atomic bomb survivors. Chest 2014:145:618-24 doi:10.1378/chest.13-1420.

10 Veronesi G, Bellomi M, Veronesi U, et al. Role of positron emission tomography scanning in the management of lung nodules detected at baseline computed tomography screening. Ann Thorac Surg 2007;84: 959-65, discussion 965-6. doi:10.1016/j.athoracsur.2007.04.058.

11 Veronesi G, Bellomi M, Mulshine JL, et al. Lung cancer screening with low-dose computed tomography: a non-invasive diagnostic protocol for baseline lung nodules. Lung Cancer 2008;61:340-9. doi:10.1016/j. lungcan.2008.01.001.

12 Radimetrics Enterprise Platform: dose management solution. Bayer HealthCare. 2014. https://www.radiologysolutions.bayer.com/ products/ct/dosemanagement/rep/.

13 Christy M, Eckerman KF. Specific absorbed fractions of energy at various ages from internal photon sources. 1987. http://citeseerx.ist. psu.edu/viewdoc/download?doi=10.1.1.453.354\&rep=rep1\&type=pdf

14 International Commission on Radiological Protection. The 2007 Recommendations of the International Commission on Radiological Protection. ICRP publication 103. Ann ICRP 2007:37:1-332.

15 Icrp. Radiation dose to patients from radiopharmaceuticals Addendum 3 to ICRP Publication 53. ICRP Publication 106. Approved by the Commission in October 2007. Ann ICRP 2008;38:1-197.
16 Mettler FA Jr, , Huda W, Yoshizumi TT, Mahesh M. Effective doses in radiology and diagnostic nuclear medicine: a catalog. Radiology 2008:248:254-63. doi:10.1148/radiol.2481071451.

17 Osei EK, Darko J. A survey of organ equivalent and effective doses from diagnostic radiology procedures. ISRN Radiol 2012;2013:204346.

18 United Nations Scientific Committee on the Effects of Atomic Radiation. UNSCEAR 2008 report to the General Assembly. Sources and effects of ionizing radiation. United Nations, 2010.

19 National Council on Radiation Protection and Measurements. Ionizing radiation exposure of the population of the United States. 2009. NCRP report 160.

20 Tubiana M. Dose-effect relationship and estimation of the carcinogenic effects of low doses of ionizing radiation: the joint report of the Académie des Sciences (Paris) and of the Académie Nationale de Médecine. Int J Radiat Oncol Biol Phys 2005;63:317-9. doi:10.1016/j.ijrobp.2005.06.013

21 Brooks AL, Dauer LT. Advances in radiation biology: effect on nuclear medicine. Semin Nucl Med 2014;44:179-86. doi:10.1053/j. semnuclmed.2014.03.004

22 Hendee WR. International Organization for Medical Physics. International organization for medical physics. Policy statement of the international organization for medical physics. Radiology 2013;267:326-7. doi:10.1148/radiol.13130567.

23 Uncertainty in risk assessment: Position of the health physics society. Health physics society website. 2013. http://hps.org/documents/ riskassessment_ps008-2.pdf.

24 Radiation risk in perspective: Position of the health physics society. Health physics society website. 2010. https://hps.org/documents/ radiationrisk.pdf.

25 Scott BR. Low-dose radiation risk extrapolation fallacy associated with the linear-no-threshold model. Hum Exp Toxicol 2008;27:163-8. doi:10.1177/0960327107083410.

26 McCollough CH, Christner JA, Kofler JM. How effective is effective dose as a predictor of radiation risk?AIR Am J Roentgenol 2010;194:890-6. doi:10.2214/AJR.09.4179.

27 Harrison JD, Balonov M, Martin CJ, et al. Use of effective dose. Ann ICRP 2016;45(Suppl):215-24. doi:10.1177/0146645316634566.

28 American College of Radiology. Lung CT Screening Reporting and Data System (Lung-RADS). https://www.acr.org/Quality-Safety/Resources/ LungRADS.

29 Larke FJ, Kruger RL, Cagnon CH, et al. Estimated radiation dose associated with low-dose chest CT of average-size participants in the National Lung Screening Trial. AJR Am J Roentgenol 2011;197:1165-9. doi:10.2214/AJR.11.6533.

30 Mascalchi M, Mazzoni LN, Falchini M, et al. Dose exposure in the ITALUNG trial of lung cancer screening with low-dose CT. Br J Radiol 2012;85:1134-9. doi:10.1259/bjr/20711289.

31 Maisonneuve P, Bagnardi V, Bellomi M, et al. Lung cancer risk prediction to select smokers for screening CT--a model based on the Italian COSMOS trial. Cancer Prev Res (Phila) 2011;4:1778-89. doi:10.1158/1940-6207.CAPR-11-0026.

32 Tammemagi MC, Lam S. Screening for lung cancer using low dose computed tomography. BMJ 2014;348:g2253. doi:10.1136/bmj.g2253.

33 Yamada Y, Jinzaki M, Tanami Y, et al. Model-based iterative reconstruction technique for ultralow-dose computed tomography of the lung: a pilot study. Invest Radiol 2012;47:482-9. doi:10.1097/ RLI.0b013e3182562a89.

34 Mathieu KB, Ai H, Fox PS, et al. Radiation dose reduction for CT lung cancer screening using ASIR and MBIR: a phantom study. J Appl Clin Med Phys 2014;15:4515.

35 den Harder AM, Willemink MJ, de Ruiter QMB, et al. Achievable dose reduction using iterative reconstruction for chest computed tomography: A systematic review. Eur J Radiol 2015;84:2307-13. doi:10.1016/j.ejrad.2015.07.011.

36 Rampinelli C, Origgi D, Vecchi V, et al. Ultra-low-dose CT with model-based iterative reconstruction (MBIR): detection of ground-glass nodules in an anthropomorphic phantom study. Radiol Med 2015;120:611-7. doi:10.1007/s11547-015-0505-5.

37 Rampinelli C, Preda L, Maniglio M, et al. Extrapulmonary malignancies detected at lung cancer screening. Radiology 2011;261:293-9. doi:10.1148/radiol.11102231.

38 van de Wiel JC, Wang Y, Xu DM, et al. NELSON study group. Neglectable benefit of searching for incidental findings in the Dutch-Belgian lung cancer screening trial (NELSON) using low-dose multidetector CT. Eur Radiol 2007:17:1474-82. doi:10.1007/ s00330-006-0532-7.

Appendix 1: Supplementary material Appendix 2: Web tables 